\title{
TRANSVAAL
}

\section{QUELQUES RAPPORTS SANITAIRES A PROPOS DE LA GUERRE SUD-AFRICAINE}

Les périodiques de médecine militaire de ces derniers mois contiennent un grand nombre de rapports sur la guerre du Transvaal, en particulier sur l'organisation sanitaire des deux armées, sur l'état sanitaire des troupes, sur le caractère général des plaies observées.

Après le rapport très louangeux de Sir W. Mac-Cormac sur l'organisation du service de santé anglais, - l'éminent chirurgien anglais a tenu à être optimiste, - après le rapport de M. Treves, moins enthousiaste surtout en ce qui touche aux secours volontaires, - on se souvient de son appréciation sur les « voluntary nurses », - est venu le rapport très sombre de $M$. W. BurdettCoutts dont les journaux politiques ont suffisamment entretenu leurs lecteurs pour que nous soyons dispensé d'y revenir. Peut-être l'autorité sanitaire anglaise en conclura-t-elle entre autres qu'à - côté de la réorganisation des services officiels, les services de la Croix-Rouge en temps de guerre ne sont pas question négligeable et que pour pouvoir compter sur eux il importe de disposer d'une organisation sanitaire volontaire dùment préparée en temps de paix. Elle a fait l'expérience un peu dure de ce que valent les services volontaires improvisés au dernier moment avec des éléments pris au hasard, et il n'est pas probable qu'elle y revienne à l'avenir.

Il y a fort longtemps que les sociétés de la Croix-Rouge, admirablement organisées dans d'autres pays d'Europe, regardaient avec étonnement du côté de l'Angleterre, ne s'expliquant pas l'indifférence et la froideur de ce pays à l'égard de la plus grande cuvre humanitaire de ce siècle ; leur étonnement n'a pas diminué lorsqu'elles ont constaté que l'organisation supérieure des services officiels, qui devaient rendre inutile toute intervention civile, existaient davantage sur le papier que dans les faits. Sans doute l'opinion publique anglaise et les victimes nombreuses de l'insuffi- 
sance des soins sur les champs de bataille, dans les hòpitaux decampagne et un peu partout dans cette mémorable guerre, obligeront-ils les services sanitaires à se préparer mieux à l'avenir; ceux-ci pourront apprendre par l'expérience des autres armées européennes, ce qu'est la Croix-Rouge, quels services on peut demander d'elle, et comment elle est organisée pour répondre d cet appel.

Le $\mathrm{D}^{\mathrm{r}}$ Clinton Dent, détaché à titre de chirurgien consultant aux. hôpitaux de l'armée dans le sud-africain y a vu passer 2 à 3000 blessés, et a pu ainsi étudier à loisir autant les questions se rattachant au fonctionnement du service de santé que les blessures par armes à feu '. Sur le premier point il réserve son opinion, ce qui ne seınble pas, étant donné le rapport très complet qu'il a présenté, faire présager une opinion très favorable. Sur le deuxième point l'auteur a constaté la bénignité relative des blessures par les armes modernes de petit calibre, du moins parmi les blessés qui ont été évacués à l'arrière. Les blessures produites par les projectiles d'artillerie, plus graves naturellement, ne représentent qu'une. faible minorité de l'ensemble des blessures, à peine le dixième du. total.

L'auteur n'a vu que très rarement des complications septiques. des plaies; faut-il l'attribuer à la nature des projectiles ou au bon état des troupes? C'est ce dont on jugera mieux lorsqu'on aura sous. les yeux les rapports provenant de l'ensemble de la campagne, en particulier en ce qui concerne-les troupes de milices envoyées. ultérieurement et mal préparées à la guerre dans ces pays.

Le transport des malades a souvent été difficile et a causé une. influence fâcheuse sur l'évolution des blessures. On fera bien de se souvenir que jamais on ne disposera de trop de moyens de transport pour les blessés et que le transport à bras, pour autant qu'il. est matériellement possible, vaut tous les transports sur roues, mềme les meilleurs.

M. Dent constate l'excellence du climat du Transvaal, climat chaud mais sec, sol sablonneux, altitude de 2000 à 5000 pieds. En. raison de ces circonstances les opérations ont pu être pratiquées dans lles conditions très favorables. La dispersion des ambulances,

1 Les blessures par fusils de petit calıbre et l'humanité dans la guerresud-africaine. Archives de méd. et de pharm. milit., 1900, no 7, p. 74. 
échelonnées sur les longues lignes de communications, a été un autre facteur de grande valeur, Il n'y a guère eu d'encombrement et les hôpitaux du Cap ont pu aisément faire face aux 1100 blessés qu'ils ont reçus par évacuations successives.

L'auteur a cherché à se faire une idée de la proportion des tués par rapport aux blessés; cette proportion aurait été d'après lui d'environ 1 à 4. Les blessures de l'abdomen ont donné une mortalité de 40 à 50 pour cent. Sur le champ de bataille le plus grand nombre des cas de mort sont résultés d'hémorrhagies. Les blessures du crâne qui n'ont pas été immédiatement mortelles ont comporté de grandes chances de guérison.

L'auteur établit une comparaison entre la guerre actuelle et la guerre franco-allemande. 4934 blessés, soit la totalité jusqu'en mars, ont donné 239 décès soit 5 pour cent; 4344 blessés du XIVmecorps d'armée allemand en ont fourni 617 , soit 14 pour cent.

Fait intéressant, constate M. Alvernhe qui a fait une excellente analyse du mémoire do M. Dent, la différence proviendrait presque uniquement des complications septiques observées en 1870; l'antisepsie jonerait donc, dans l'atténuation de la mortalité, un ròle infiniment plus important que la modification introduite dans les armements. Les Américains ont relevé, au cours de la guerre hispano-américaine un taux de mortalité sensiblement égal à celui de M. Dent, soit environ 6 pour cent.

L'opinion du Dr Treves, chirurgien consultant du corps expéditionnaire, ' sur les blessures dans la guerre actuelle, concordent dans leur ensemble avec celles du $\mathrm{D}^{\mathrm{r}}$ Dent. Comme lui il constate que le fusil de l'ancien armement, spécialement le Martini-Henry, metlait plus sùrement les hommes hors de combat que le fusil de petit calibre actuel. L'auteur ne pense pas que la formule $\mathrm{M} \mathrm{V}^{2}$ ( $M$ représentant la masse du projectile et $V$ sa vitesse), généralement acceptée comme traduisant la force vive de la balle, soit l'expression de la vérité. Tout en reconnaissant la difficulté d'apprécier la distance sur le terrain, surtout avec l'emploi de la pondre sans fumée, il croit pouvoir conclure des combats auxquels il a assisté que la distance est loin de jouer, au point de vue de la gravité des blessures, un rôle aussi important qu'on le croyait. De même les effets dits « explosifs» ne seraient pas, mème pour les projectiles 
du fusil Mauser, uniquement déterminés par la distance, des blessures failes à une très courte distance peuvent être d'une netteté parfaite, même en cas de lésion des os; cependant l'auteur n'a constaté d'effets explosifs qu'à une portée relativement courte et dans ce cas, chaque fois que le projectile était resté dans la plaie, on l'a trouvé déformé.

Parmi les blessures des armes de l'artillerie, les effets destructifs des obus à lyddite ont été, dit $M$. Treves, fortement exagérés; loutefois ces obus dégagent une épaisse fumée jaunâtre suffocante et plusieurs Boers, apportés à l'ambulance sous le coup d'une commotion produite par cet explosif, sont restés un ou deux jours insensibles, sans présenter aucune trace de lésion.

M. Treves s'étend assez longuement sur les blessures de l'abdomen; il estime que la chirurgie abdominale, n'a pas tenu ses promesses et n'a donné que des mécomptes, aussi se déclare-t-il, en principe, du moins, franchement abstentionniste. L'auteur a eu beaucoup à se louer du pansement individuel qui a rendu, dit-il, dans cette guerre des services inappréciables.

Nous extrayons, d'autre part, les quelques renseignements suivants d'un rapport de M. le $D^{r}$ Küttner, un des médecins délégués au Transvaal par la Croix-Rouge allemande '. D'accord avec les observateurs précédents, ce chirurgien constate que le petit calibre a donné, en général, des plaies moins graves que celles des anciennes armes; les combattants se sont souvent plaints de la rapidité de la guérison des blessés qui pouvaient, après peu de temps souvent, reprendre les armes. Celte opinion, dit le $D^{\text {r }}$ Küttner, semble en contradiction aver le fait que la proportion des blessés et des morts est de 1 pour 1, autrement dit égale. On voit que le $\mathrm{D}^{\mathrm{r}}$ Küttner qui a travaillé près des champs de bataille a, à cet égard, une opinion bien différente de celle de M. Dent qui fonctionnait à l'arrière et parlait d'une proportion de 1 pour 4 seulement. M. Kültner estime que cette proportion énorme de mortalité provient de ce que les Boers tirent avec une telle précision que les blessures de la tête ont élé très fréquentes et ont été la cause du grand nombre des cas de mort chez les Anglais, comme du reste aussi chez les Boers, ceux-ci ayant 'habitude de se retrancher de telle manière que les projectiles qui

- Deutsche Militärärztliche Zeitschrift, 1900, $\mathrm{n}^{\circ}$ 6, p. 343 . 
les atleignaient ne pouvaient guère toucher autre part qu'à la tête qui était seule visible. Pour le mème motif, la majorité des plaies chez les Boers ont été produites par les projectiles de l'artillerie.

L'auteur mentionne quelques plaies particulièrement intéressantes : ainsi un Boer tirant à genoux, reçut par derrière et de haut une balle qui lui entra dans le dos pour ressortir à l'aine, rentrer à un centimètre plus bas dans la cuisse, ressortir sous le genou, rentrer dans le mollet pour ressortir enfin en avant du péroné, avec blessure de cel os. Le malade guéril de ses six plaies. après avoir eu un peu de réaction péritonitique.

Un autre blessé reçul un coup de baïonnette (soit dit en passant les blessures par armes blanches ont élé très rares dans cetle guerre) en dedans de l'omoplate, l'arme traversa tout le thorax et vint ressortir en avant près de la tête de l'humérus. Entrẻe jusqu'd la garde, la baïonnette s'était cassée en cet endroit, formant une large plaie déchirée. Le blessé ne trouva d'autre moyen pour se désempaler, que de frapper avec une pierre contre la pointe de la baïonnette jusqu'à ce qu'elle fút suffisamment sortie en arrière pour lui permettre de l'extraire aver l'autre main. Chose curieuse, il n'y eut ni blessure artérielle ni blessure nerveuse et le malade guérit sans complications.

Encore un mot à propos de ce rapporl: M. Küttner n'a pas vu de blessures produites par la balle dum-dum; cependant il constate qu'on doit avoir tiré avec ce projectile. Sur plusieurs soldats tués à la bataille de Maggersfontein, on a trouvé, en effet, des projectiles du mod. $\mathrm{n}^{\circ} 10$ dans le sac à cartouches de gauche, tandis que celui de droite contenait des balles ordinaires à enveloppe normale. Comme l'on a tiré surtout à de grandes distances, les soldats anglais auront fait l'expérience que le projectile ordinaire convenait mieux, vu sa portée et « lorsqu'on en est arrivé au combat à courtes distances, dit le $\mathrm{D}^{\mathrm{r}}$ Küttner, ils n'ont le plus souvent plus tiré bien longlemps »; d’où la grande rareté des blessures par balle dum-dum.

Le résultat négatif des observations de $M$. Küttner à cet égard est conforme à celles de Sir W. Mac-Cormac; dans le rapport qu'il a fourni sur la guerre sud-africaine, l'éminent chirurgien anglais déclare qu'il n'a vu aucune plaie imputableà la balle dum-dum ou à la balle «soft nosed ». Il est bon de relever ces faits puisqu'on a beaucoup parlé de l'empłoi de ces projectiles à propos de la guerre actuelle. 
Le correspondant militaire sud-africain du Medical Record ${ }^{1}$ donne le relevé des pertes de l'armée anglaise jusqu'à la prise de Prétoria, en voici le détail :

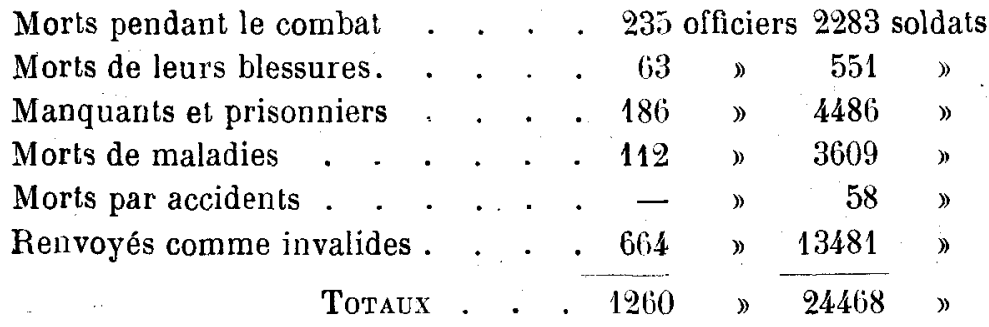

En tout donc 25,728 hommes, sans compter les malades et blessés qui se trouvaient à cette époque dans les hôpitaux anglais du sud de l'Afrique. Done en huil mois l'armée anglaise a perdu $12 \frac{1}{2} \%$ de son effectif. Les officiers; qui comptent pour moins de 1 pour cent dans l'armée, ont succombé dans la proportion de 1 pour 10, pendant l'action, et de 1 pour 9 , à la suite de blessures; ce qui prouve que cette catégorie n'a été privilégiée ni pendant le combat ni dans les soins à l'ambulance.

La proportion des officiers morts de maladie, comparativement à celle des soldats, est de 1 pour 30, et, sur ce point, l'auteur estime qu'il faut attribuer cel avantage très grand du côté des officiers plutôt aux ressources personnelles de ces derniers qu'aux conditions meilleures qui leur ont été faites quant au logement el à la nourriture. Dans les marches, officiers et troupe ont partagé le même genre de vie, mais dans les haltes les officiers ont le plus souvent pu s'accoràer un certain confort. Plus de $\% / \mathrm{s}$ des pertes figurent sur les listes des manquants ou prisonniers et des invalides renvoyés dans leurs foyers; il y a donc lieu de ne pas ranger ces catégories parmi les pertes définitives, puisque le plus grand nombre des hommes qui en dépendent conserveront ou retrouveront santé et validité. Il semble à ce propos que les prisonniers anglais retenus par les Boers unt été généralement bien traités, malgré l'extrême difficulté des circonstances, et il y aura lieu, tout au moins, de leur en savoir gré et de leur tenir compte de ce fait lors du règlement final de cetle malheureuse guerre.

La morbidité et la mortalité dans la guerre sud-africaine ont

I Voir $n^{\circ}$ du 7 juillet, p. 29. 
été, comme on le sait, assez fortes, très fortes même. On doit en chercher la cause soit dans le climat lu pays, soit dans les influences saisonnières, soit dans l'entretien des troupes. On sait que les opinions sont partagées quant à ce dernier facteur, mais s'il faut admettre, comme l'ont constaté les rapports de Sir W. MacCormac, de M. Dent, de M. Treves et d'autres, que l'alimentation de la troupe a été assurée d'une manière absolument normale par l'intendance militaire, il est hors de doute aussi que les rapports de M. Burdett-Coutts sont absolument véridiques et point exagérés.

Parmi les causes locales, les ouragans de poussière ont causé passablement de maladies dans l'armée anglaise : affections de la gorge, des yęux, infections diverses. Les alternances de pluies torrentielles et de vents secs, les différences considérables de température dans les 24 heures, n'étaient pas faites non plus pour diminuer les dangers d'infection. La fièvre typhoïde a sévi avec une certaine intensité dans les troupes anglaises. Si l'on considère la proportion des malades par rapport au le nombre total des hommes, on ne peut parler d'une forte épidémie, disent les uns, tandis que d'autres donnent le chiffre de morbidité comme énorme.

On voit qu'il est difficile jusqu'à plus amples informations de se faire une opinion. Nous penchons toutefois du côté des pessimistes à la lecture de certains rappor's qui nous viennent du théâtre des hostilités. Ainsi M. Fripp, dans une lettre au British Medical Journal ${ }^{1}$, dit qu'à l'époque où il écrivait, il y avait près de 2000 typhoïdiques rien qu'à Blœmfontein. On sait d'autre part qu'à Kimberley, à Mafeking et à Ladysmith l'épidémie a été violente pendant le siège de ces places.

Le Journal of Tropical Medecine dit aussi dans son numéro du 15 juin :

Nous avons recherché récemment le nombre des décès par fièvre typhoïde dans l'armée du sud de l'Afrique, dans la liste officielle des morts par maladie; nous n'en trouvons pas moins de 100 entre le 30 mai et le 6 juin, et, sur ce nombre, 23 à la seule date du 4 juin. 'L'anteur en conclut que l'épidémie est forte et très répandue aussi, les décès provenant d'un grand nombre de stations diverses.

Pendant l'épidémie typhoïdique qui a sévi dans certains camps de l'armée américaine, en 1898 , le $D^{r}$ Vaughan a constaté que la

1 Typhoïd fever among the british troops in South Africa. - Medical. Record, 21 juillet, p. 98. 
propagation s'est faite, sans doute, ell grande partie par les moustiques et par la poussière; ces deux causes d'infection sont plus que vraisemblables dans le sud-africain où, en certaines saisons, ellesconstituent un inconvénient plus accentué peut-être que dans. aucune autre partie du monde.

On se souvient des inoculations préventives contre la fièvre typhoïde pratiquées sur quelques milliers de soldats avant leur départ de l'Angleterre ; ces inoculations qui ont fort ennuyé la troupe à ce moment, ont-elles du moins porté leur fruit dans la suite? Il ne semble guère; en tous les cas nombre d'entre les inoculés sont morts de fièvres entériques; s'est-il agi de fièvre typhoïde ou de malaria ou typho-malaria? C'est ce qui ressortira des observations qui ont été dit-on très soigneusement relevées dans les hôpitaux sud-africains. Voici, du reste, à ce sujet l'opinion de Sir W. Mac-Cormac: «On ne peut, dit-il, émettre d'opinion sur ce point avant d'avoir accumulé un nombre suffisant d'observations. statistiques. D'après les faits constatés jusqu'ici, il semble que les sujets inoculés aient présenté moins de disposition à contracter la. maladie, ou bien lorsqu'ils ont été atteints, que leur affection ait: été plus bénigne; mais la question reste à l'examen. 》

Les nouvelles venues du côté des Boers sont, au point de vue qui nous occupe, peu détaillées. La fièvre typhoïde a sévi aussi chez eux avec une grande intensité et leur prisonniers semblent l'avoir apportée avec eux dans différentes localités d'internement où cette maladie n'avait pas paru jusque-là ; mais les conditions de ces internements ont donné lieu d'autre part à des récriminations violentes. et les infections secondaires ont joué de ce chef un rôle considérable, a-t-on affirmé, dans l'extension du mal.

La morbidité et la mortalité élevées de la fièvre typhoïde dans celte campagne ne sont du reste pas chose exceptionnelle; cette maladie a été la compagne habituelle de toutes les guerres continentales et coloniales de ce siècle. ${ }^{1}$

Sans remonter très haut, voici quelques chiffres instructifs à ce sujet : Pendant la guerre de Sécession; de 1861 à 1866, l'armée américaine a compté un effectif moyen de 431,000 hommes; le chiffre des fièvres typhoïdes et typho-malariennes a atteint plus de $13 \bar{i}, 000$

I Lr H. Vincent. Fréquence de la fièvre typhoïde dans les guerres modernes. Archives de méd. et de pharm. milit., no 8, 1900, p. 124. 
cas, avec une mortalité de plus de 31,000 décès. Pendant la guerre franco-allemande, la statistique obituaire de l'armée allemande donne plus de 7000 morts de feevre typhoïde pour un effectif de 815,000 hommes. Pendant le siège de Paris la mortalité par typhoïde s'éleva à 40 pour cent des malades. Dans la guerre turcorusse, en 28 mois, l'armée du Danube, forte de 592,000 hommes, eut 25,000 cas de fièvre typhoïde avec plus de 7000 décès, tandis que les pertes par le feu, malgré de sanglants combats, n'était que de 4673 tués. Dans l'armée du Caucase, de même, il y eut $1869^{\circ}$ décès par armes à feu, pour plus de 24,000 cas de fièvre typhoïdeet près de 9000 décès. Pendant la campagne de Bosnie-Herzégovine, sur 2233 décès survenus pendant cette courte guerre, il y eut 944 morts par fièvre typhoïde et seulement 277 décès résultant des combats. Pendant la campagne des Anglais en Egypte, la morbidité typhoïdique s'éleva au .chiffre considérable de 421,4 pour mille hommes de troupe, et, à peu près à la même époque, l'armée.française, pendant la guerre de Tunisie, comptait 4200 cas de fièvre typhoïde sur un effectif de 20,000 hommes, avec 1039 décès. Nous pourrions continuer celte liste lugubre à travers toutes les autres guerres de ces dernières années; ce sont toujours à peu près les mêmes proportions, aussi bien dans la guerre sino-japonaise que dans la guerre hispano-américaine, de même que dans la guerre actuelle du Transvaal.

L'origine de ces épidémies des camps a été fort bien définie par le $\mathrm{D}^{\mathrm{r}}$ Simonin dans un récent mémoire ${ }^{1}$ : l'ensemble des souffrances qui naissent de la guerre, et surtout des guerres de longue durée, l'excès et la continuité des fatigues, joints le plus souvent à l'insuffisance alimentaire, produisent une véritable dyspepsie gastro-intestinale qui ne tarde pas à laisser le champ libre à la série des auto-infections des voies digestives. Cette influence de la fatigue se fait sentir sur les individus comme sur les masses armées et d'une façon souvent proportionnelle aux efforts demandés. $Q u^{\prime}$ 'on ajoute à celte cause l'action considérable de la contagion, la propagation de l'infection des groupes militaires aux milieux civils qu'ils traversent, populations le plus souvent durement éprouvées par la guerre, et l'on n'est plus étonné de l'extension énorme que prennent ces épidémies meurtrières où la fièvre typhoïde se rattache étroitement $\dot{a}$ la dysenterie et sourent au paludisme.

${ }^{\prime}$ Archiv. de méd. et de pharm. milit., $\mathrm{n}^{\circ} 8, \mathrm{p} .144$. 
La prophylaxie se déduit tout naturellement des causes, elle est la mème pour ces différentes catégories d'infection et dépend autant de l'intelligence et du zèle des chefs de troupe que de l'intendance militaire et de l'autorité sanitaire. Mais ce n'est pas à dire que nous soyons à la veille de progrès bien marqués à cet égard, et il est plus que probable que le désastre que subit actuellement l'armée anglaise au Transvaal, par son épidémie typhoïdique de plus en plus répan. due, se répètera dans les guerres futures, car l'hygiène d'une armée en temps de guerre est chose toujours plus ou moins illusoire '. Du moins convenait-il de constater, à la décharge de l'autorité militaire anglaise, que l'épidémie de typhoïde n'est pas un fait exceptionnel de la guerre sud-africaine, mais qu'elle a été la règle dans toutes les guerres de ce siècle.

Le correspondant sud-africain du Medical Record' ${ }^{2}$ a eu l'occasion de visiter une ambulance boer tombée entre les mains de lord Roberts lors de son entrée à Blœmfontein. On avait beaucoup dit que l'armée boer étail très mal fournie en moyens de secours pour les blessés; le correspondant l'avait cru lui-même, et l'avait répété après d'autres dans des articles précédents. Il reconnaît que l'ambulance trouvée à Blœmfontein était fort bien organisée, composée de voitures très confortables bien que de formes assez disparates; la seule uniformité consistait dans la présence du drapeau de la Croix-Rouge, fixé à chaque chariot. Partout de bons matelas, de bons brancards, et tout un matériel de choix organisé en entier, contrairement à ce qui est en usage dans les ambulances anglaises, pour effectuer le transport des blessés dans la position étendue.

Le même correspondant a constaté, comme d'autres du reste, avec étonnement, l'accueil très froid des Boers à l'égard des médecins venus à eux, poussés par l'élan d'une pensée généreuse, pour porter secours à leurs blessés. Il attribue cette froideur d'une

I Si nous devons assister cet hiver à une campagne en Chine. il faudra compter sur une forte épidémie typhoïđique parmi les troupes européennes. Après les inondations habituelles de ce pays, en été, l'hiver comporte une série de semaines avec vents violents et poussières intenses; a ce moment la fièvre tỵphoïde serait endémique dans une grande partie de la Chine et causerait habituellement un très grand nombre de victimes; d'où, dit-on, l'habitude des Chinois d'éviter de boire de l'eau non bouilie et l'usage qu'ils font d'infusions de the chaud.

14 avril, p. 650 . 
part à la déception causée au Transvaal par l'attitude plus que réservée de l'Europe et d'autre part à la jalousie professionnelle des médecins boers; il en donne des exemples. Ces populations rustiques qui se sont distinguées par leurs vertus civiques, la bravoure, le sang-froid, l'endurance, et par dessus tout par la dévotion absolue à leur cause, manquent évidemment de formes, d'aménité et de courtoisie ; leur rudesse est proverbiale et peut-être estelle pour beaucoup dans leurs malheurs.

Un mot toutefois, en terminant, à leur défense : on a répété à plusieurs reprises que les Boers ont tiré sur des ambulances et des hôpitaux. Sir William Thompson, chirurgien de l'hòpital irlandais, constate que ce fait a dû être, le plus souvent du moins, accidentel, car, dit-il, les drapeaux de la Croix-Rouge sur les ambulances anglaises étaient placés de manière à n'être visibles qu'à une distance de 600 mètres environ ', ce qui est absolument insuffisant. Décidément les corps sanitaires anglais ne pourront à l'avenir que gagner à affirmer de plus en plus nettement la CroixRouge dans la forme comme dans le fond.

Dr Ferrière. 